\title{
Hotspots API: A toolkit for the application of Fragment Hotspot Mapping to Structure Based Drug Discovery
}

$\underline{\text { Peter Curran }}^{\mathrm{a}}$, Chris Radoux ${ }^{\mathrm{b}}$, Mihaela Smilova ${ }^{\mathrm{c}}$, Alicia Higueruelo ${ }^{\mathrm{d}}$, Richard Sykes ${ }^{\mathrm{e}}$, Anthony Bradley ${ }^{\mathrm{f}}$, Will Pitt ${ }^{\mathrm{g}}$, David Spring ${ }^{\mathrm{h}}$, Tom Blundell', Jason Cole ${ }^{\mathrm{j}}$

a CCDC, Cambridge, UK / Department of Chemistry, University of Cambridge, UK, pcurran@ccdc.cam.ac.uk

${ }^{b}$ EBI, Cambridge, UK, cradoux@ebi.ac.uk

c University of Oxford, UK, mihaela.smilova@linacre.ox.ac.uk

d Charles River Laboratories, UK, Alicia.Higueruelo@crl.com

e CCDC, Cambridge, UK, sykes@ccdc.cam.ac.uk

${ }^{f}$ Exscientia, UK, anthony.richard.bradley@gmail.com

g UCB Pharma, Slough, UK, Will.Pitt@ucb.com

h Department of Chemistry, University of Cambridge, UK, spring@ch.cam.ac.uk

' Department of Biochemistry, University of Cambridge, UK, tom@cryst.bioc.cam.ac.uk

JCCDC, Cambridge, UK, cole@ccdc.cam.ac.uk

\begin{abstract}
:
Small molecule binding hotspots are regions within a protein pocket that contribute a disproportionately large amount to the overall binding energy. Consequently, fragments which bind to hotspots are anchored in place, providing an energetic foothold from which structure-based methods can efficiently guide designs towards ligand efficient lead molecules. Radoux et al. (J. Med. Chem., 2016, 59 (9), pp 4314-4325) developed a fast, knowledge-based method to characterise the location and critical intermolecular interactions in these regions, using data derived from the Cambridge Structural Database (CSD). Since this publication, we have continued to develop this algorithm and design workflows to utilise these valuable insights. To enable this, we have recently released the Hotspots API, a Python API built upon the CSD Python API (https://github.com/prcurran/hotspots). This talk will outline some exemplar use cases, including tractability assessment, assignment of docking constraints and generation of pharmacophoric models. We also outline broader plans to use this tool for the design of small molecule screening libraries.
\end{abstract}

